\title{
CASSINI-HUYGENS
}

\section{Saturn in the infrared}

The reason I am so partial to this image is that it shows a completely different view of Saturn than anything we ever saw before the Cassini spacecraft went into orbit. The Visual and Infrared Mapping Spectrometer (VIMS) observes at 352 different wavelengths between 0.3 and $5.1 \mu \mathrm{m}$, enabling different processes at different depths to all be viewed in the same false-colour image. At $2.3 \mu \mathrm{m}$ (shown in blue), the icy ring particles are highly reflecting, while the methane gas in the atmosphere of Saturn strongly absorbs the sunlight, causing the planet to appear dark. At $3.0 \mu \mathrm{m}$ (shown in green), the situation is reversed: water ice in the rings is strongly absorbing, whereas the planet's sunlit hemisphere is bright. As a result the rings appear blue, while the sunlit side of Saturn is greenish-yellow in colour.

Within the rings, the most opaque parts appear dark, while the more translucent regions are brighter. In particular, the opaque, normally bright $B$ ring appears here as a broad, dark band separating the brighter $\mathrm{A}$ (outer) and $\mathrm{C}$ (inner) rings. At $5.1 \mu \mathrm{m}$ (shown in red), reflected sunlight is weak and as a result the light from the planet is dominated by thermal radiation that wells up from

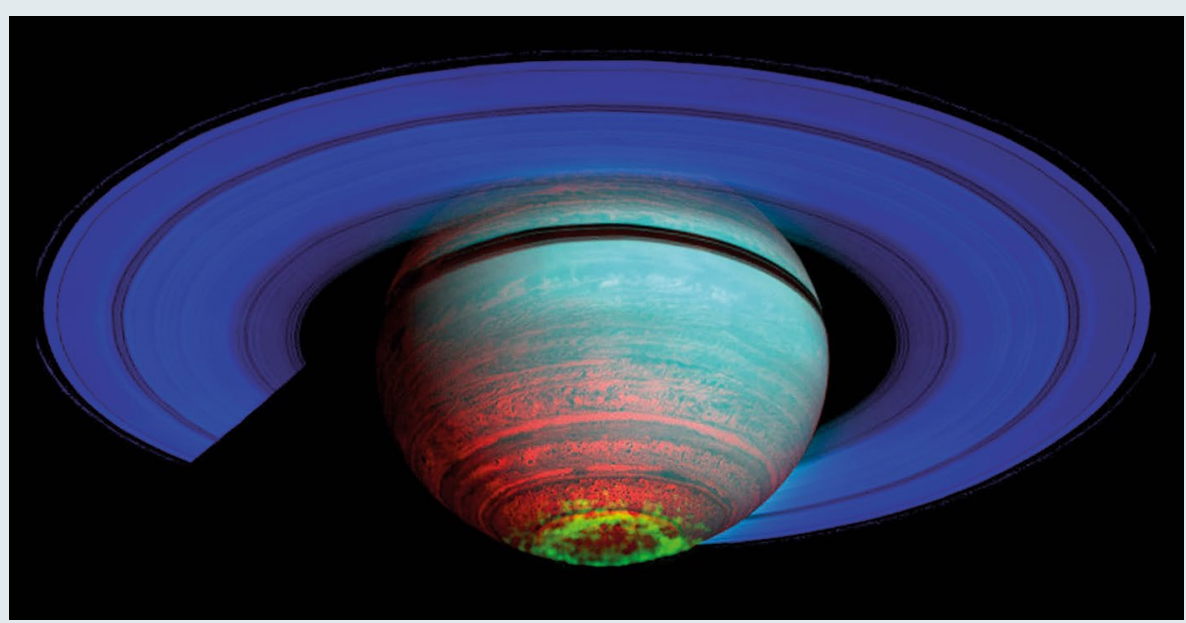

Image credit: NASA \JPL\University of Arizona \University of Leicester

the planet's deep atmosphere. Variable amounts of clouds in the planet's upper atmosphere block the thermal radiation, leading to a speckled and banded appearance, which is always changing due to the planet's strong winds. Last but not least, one can view the bright green emission of the southern aurora generated as a result of precipitating particles spiralling along Saturn's magnetic field lines near the south pole, which for me is the cherry on the cake since my instrument measures the magnetic field lines.

Michele K. Dougherty

The Blackett Laboratory, Imperial College London, Prince Consort Road, London SW7 2AZ, UK. e-mail:m.dougherty@imperial.ac.uk

Published online: 5 September 2017 DOI: 10.1038/s41550-017-0245-7 